\title{
Recurrence of Panic Attacks after Influenza Vaccination: Two Case Reports
}

\author{
Han-Joon Kim, Sang-Won Jeon, Ho-Kyoung Yoon \\ Department of Psychiatry, Korea University Ansan Hospital, Korea University College of Medicine, Ansan, Korea
}

\begin{abstract}
Human influenza is a contagious respiratory illness caused by the influenza virus. The influenza vaccination is recommended annually, but several adverse effects related to allergic reactions have been reported. Panic attacks are also known to occur, but no case of a panic attack adverse effect has been reported in South Korea. We present two cases of panic disorder patients whose symptoms were aggravated by the influenza vaccination. We assumed that dysregulation of T-lymphocytes in panic disorder patients could have a role in activating various kinds of cytokines and chemokines, which then can lead to panic attack aggravation.
\end{abstract}

KEY WORDS: Human influenza; Influenza vaccines; Panic attack; Adverse effects.

\section{INTRODUCTION}

Influenza is a contagious respiratory illness caused by the influenza viruses. Since the 1940s, inactivated influenza vaccines have been introduced, ${ }^{1)}$ and they decreased the mortality of influenza by $41 \%{ }^{2)}$ However, there were also reports on the side effects of the influenza vaccination, including injection site pain, swelling, fatigue, fever, and allergic reactions, including anaphylaxis. ${ }^{3)}$ There were also reports on panic attacks following influenza vaccination. ${ }^{4}$ However, the mechanisms of a panic attack occurrence after the vaccination have been not revealed, and few cases have been reported globally so far. We present the cases of two panic disorder patients who suffered panic attacks after receiving the influenza vaccine in South Korea.

\section{CASE}

\section{Case 1}

A 51 year-old woman who had been treated for a panic disorder since 2004 visited our outpatient clinic and was admitted with symptoms of chest tightness, palpitation,

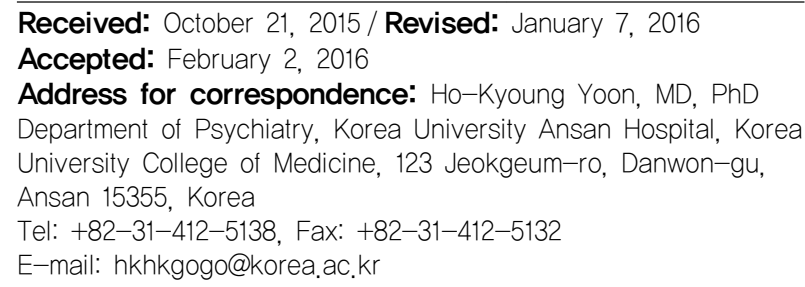

A 45 year-old man diagnosed with a panic disorder in April 2014 visited our outpatient clinic in June of 2014. He was suffering from sudden chest discomfort, sweating, and light-headedness. He had experienced the symptoms for more than 10 minutes, but negative test results were found on his EKG, chest X-ray, and blood tests. He had severe anticipatory anxiety, and we decided him to admit in July of 2014. He took paroxetine CR $37.5 \mathrm{mg}$ /day, trazober of 2014. She underwent a chest X-ray and elecwere checked, but no specific abnormality was found. She was diagnosed with a panic disorder, and took mirtazapine $7.5 \mathrm{mg} /$ day, escitalopram $5 \mathrm{mg} /$ day, and lorazepam 1 $\mathrm{mg}$ /day. She was admitted for 2 weeks and discharged when her symptoms were alleviated. She visited our outpatient clinic regularly, and was stable on her medications. In November 2014, her feelings of choking, palpitation, chest tightness, and tingling senses were aggravated. She had no history of allergic reaction, but was worried about the influenza vaccination, which she had received the day before. She said her symptoms waxed and waned, and lasted over 24 hours. We recommended her to visit the emergency room, but she visited outpatient clinic several days after when her symptoms spontaneously resolved. She took an additional alprazolam $0.5 \mathrm{mg} /$ day, and sustained treatment in the outpatient clinic without extra panic attacks or additional admissions. 
done $25 \mathrm{mg}$ /day, alprazolam $0.75 \mathrm{mg} /$ day. After one month of treatment, he felt his symptoms had improved, and was discharged. But, in November of 2014, he experienced aggravated symptoms for several hours, directly after he received the influenza vaccination. He had no history of allergic reaction, and voluntarily visited the outpatient clinic, and was admitted for panic attack aggravation. In the early stage of admission, he underwent panic attacks once a week, despite of negative test results on EKG and blood tests. After he was prescribed with additional tianeptine $25 \mathrm{mg} /$ day, his symptoms improved, and he was discharged one month later, after being panic attack-free for a week.

\section{DISCUSSION}

Both patients in our case report gave informed consent for the publication of case report. They received a trivalent inactivated influenza vaccine, including type A antigens (California/7/2009 [H1N1], Victoria/361/2011 [H3N2]) and a type B antigen (Massachusetts/02/2012). Adjuvants, preservatives, or stabilizers, such as formaldehyde, gentamycin, deoxycholic acid were also included in vaccine, but thimerosal was not included. In general, there are immunologic and non-immunologic mechanisms that cause the side effects of vaccination. The fear of injection, inappropriate injection site, and poor hygiene of healthcare workers can be related to the non-immunologic factors, ${ }^{5)}$ but our case, neither patient had a history of previous injection fear. Both of patients had been taken annual vaccination of influenza for more than 10 years, but did not experience substantial side effect of vaccination or have anxiety of being vaccinated. The patients were vaccinated by well-trained healthcare workers in hospitals. We could assume that our patients' side effects were caused directly by the vaccine itself. Therefore, we assumed that our patients' side effects were panic attack aggravation, despite of the limitation that we retrospectively examined our patients' symptoms, and there could be difficulty of attributing panic attack aggravation purely on vaccination. We considered that the differences of vaccination's composition may cause the immunologic reaction in the patients who received the influenza vaccination in the November 2014

The mechanism of a panic attack following vaccination is not fully explained, but the immunological change might have role in this process. Currently, only split vaccines derived from the envelope of virus are used in South Korea. ${ }^{3)}$ Various types of immunoglobulin react with the vaccine in our bodies, and these antigen-antibody reactions are sup- ported by the T and B lymphocytes. CD4, CD8 T-lymphocyte activated by the vaccine regulate the B-lymphocyte, which differentiates into its plasma cell and memory cell roles in recognizing and remembering the virus, respectively. However, an extremely activated immune system can result in a hypersensitive reaction. ${ }^{6}$

Panic disorders are also known to have immunological pathophysiology. Previous studies have led to an increased recognition that pro-inflammatory cytokines could enhance the activity of enzymes related to the degradation of neurotransmitters. ${ }^{7,8)}$ A broad spectrum of cytokine abnormalities, which suggest a generalized inflammatory state, have been shown to be present in individuals with panic disorder. ${ }^{9)}$ Several studies have shown that stress can activate or inhibit the immune system, thus modulating the number of T-lymphocytes. ${ }^{10,11)}$ Preliminary study also showed that T-lymphocytes have high-affinity cholecystokinin receptors, which induce symptoms comparable to those of panic attack. ${ }^{12)}$ There was also a study that revealed that increased CD3, CD4, and CD8 T-lymphocyte, and decreased CD 19 B-lymphocyte were found in panic disorder patients with pharmacological treatment. ${ }^{13)}$ With this information, we assumed that the Tlymphocyte could mediate the panic attack following the vaccination. Through the antiviral process, various kind of cytokines and chemokines regulated by abnormal T-lymphocytes in panic disorder patients might have roles in the aggravation of panic attacks. ${ }^{6}$

However, other studies have shown that there was no difference in T-lymphocyte subsets between panic disorder patients and control groups. ${ }^{14,15)}$ There should be more consideration for these results, and the precise molecular mechanisms of antiviral immunologic pathways should be studied further.

To our knowledge, this is the first case report of influenza vaccine-associated panic attacks. There were common immunological factors shown in the vaccination and panic attack, which are mainly explained by T-lymphocyte dysregulation. However, few studies have been performed or report on these potential mechanisms. Further studies are required to understand the precise mechanism of a panic attack induced by vaccination. Although there are many different arguments, we hope our report will lead clinicians to monitor their patients for the vaccination's potential adverse effect.

\section{REFERENCES}

1. Gerdil C. The annual production cycle for influenza vaccine. Vaccine 2003;21:1776-1779. 
2. Ahmed AE, Nicholson KG, Nguyen-Van-Tam JS. Reduction in mortality associated with influenza vaccine during 1989-90 epidemic. Lancet 1995;346:591-595.

3. Cheong HJ. Safety of influenza vaccine. J Korean Med Assoc 2007;50:267-273.

4. Moro PL, Broder K, Zheteyeva Y, Revzina N, Tepper N, Kissin D, et al. Adverse events following administration to pregnant women of influenza A (H1N1) 2009 monovalent vaccine reported to the Vaccine Adverse Event Reporting System. Am J Obstet Gynecol 2011;205:473.e1-e9.

5. Siegrist CA. Mechanisms underlying adverse reactions to vaccines. J Comp Pathol 2007;137 Suppl 1:S46-S50.

6. Cho $\mathrm{H}$, Kang $\mathrm{H}$. Host immune responses against type a influenza viruses. J Bacteriol Virol 2014;44:133-139.

7. Myint AM, Kim YK. Cytokine-serotonin interaction through IDO: a neurodegeneration hypothesis of depression. Med Hypotheses 2003;61:519-525.

8. Myint AM, Kim YK. Network beyond IDO in psychiatric disorders: revisiting neurodegeneration hypothesis. Prog Neuropsychopharmacol Biol Psychiatry 2014:48:304-313.

9. Hoge EA, Brandstetter K, Moshier S, Pollack MH, Wong KK, Simon NM. Broad spectrum of cytokine abnormalities in panic disorder and posttraumatic stress disorder. Depress
Anxiety 2009;26:447-455.

10. Andreoli A, Keller SE, Rabaeus M, Zaugg L, Garrone G, Taban C. Immunity, major depression, and panic disorder comorbidity. Biol Psychiatry 1992;31:896-908.

11. Keller SE, Weiss JM, Schleifer SJ, Miller NE, Stein M. Suppression of immunity by stress: effect of a graded series of stressors on lymphocyte stimulation in the rat. Science 1981;213:1397-1400.

12. Akiyoshi J, Isogawa $\mathrm{K}$, Tsutsumi T, Kasturagi S, Kohno K, Furuta M, et al. Cholecystokinin tetrapeptide-induced calcium mobilization in $T$ cells of patients with panic disorder, major depression, or schizophrenia. Biol Psychiatry 1997; 42:151-154.

13. Schleifer SJ, Keller SE, Bartlett JA. Panic disorder and immunity: few effects on circulating lymphocytes, mitogen response, and NK cell activity. Brain Behav Immun 2002; 16:698-705.

14. Park JE, Kim SW, Park Q, Jeong DU, Yu BH. Lymphocyte subsets and mood states in panic disorder patients. $J$ Korean Med Sci 2005;20:215-219.

15. Kim YR, Park Q, Yu BH. Changes in lymphocyte subsets after short-term pharmacotherapy in patients with panic disorder. Psychiatry Res 2004;128:183-190. 